\title{
Development of serious computer game based training module and its integration into working at heights mine site induction Part I
}

\author{
Phillip Stothard*, School of Mining Engineering, UNSW, Sydney, NSW 2052, Australia \\ *Corresponding author, email Phillip.stothard@curtin.edu.au \\ Anton van den Hengel, School of Computer Science, University of Adelaide, Adelaide, SA 5005, Australia
}

\begin{abstract}
This paper reports the findings of a collaborative project that developed and demonstrated a serious computer game (SCG) based simulation training module for mine site inductions. It is the first of two papers. The project was collaboration between the University of New South Wales, the University of Adelaide, BHPB Olympic Dam Expansion, Resources and Engineering Skills Alliance, TAFESA and Skills DMC. The project was a pilot aimed at improving mine site inductions by developing a prototype SCG for trainers to incorporate into their regular training activities. The outcome was a high quality generic SCG that provides an interactive visualisation of an Australian mine site operation under construction. The conclusions and recommendations derived from this part of the study are summarised and discussion on the improvement of the SCG based simulation module is presented and some recommendations made. Scientific testing of the module and its site deployment are presented in the subsequent paper.
\end{abstract}

\section{Keywords: Mine safety, Simulation, Serious games}

Introduction

This is the first of two papers that review the findings of a collaborative project that developed and demonstrated a serious computer game (SCG) based simulation training module for mine site inductions. The paper discusses the project from conception through to prototype testing prior to site deployment.

The project was collaboration between the University of New South Wales (UNSW), School of Mining Engineering, the University of Adelaide, Australian Centre for Visual Technologies (ACVT), a major mining company, BHPB Olympic Dam Expansion, government training advisors Resources and Engineering Skills Alliance, TAFESA and Skills DMC. The project was a pilot study aimed at improving mine site inductions for the hard rock mining industry of South Australia by developing a prototype SCG for trainers to incorporate into their regular training activities that would lead to an improved understanding of mine operations and safety.

An objective was to build a generic pilot module that was used to assess the suitability of SCG technologies to conduct training tasks within the mining industry. It was expected that the SCG module would also have application to the wider construction industry and other heavy industries such as oil and gas. Invaluable input was provided by the industry collaborators via the steering committee, access to a mine site, key personnel and contract trainers.

The subject area 'working at heights' was chosen due to its importance as a safety issue across all mine sites. Substantial effort, documentation review and mining industry input was spent on building the five Working at Heights sub modules: Correctly Erected Scaffolding, Incomplete Scaffolding, Open Excavation, Light Globe Change using Ladders and Elevated Work Platform.

The outcome of the research was a high quality generic SCG that provides an interactive visualisation of an Australian mine site operation that was under construction. The visualisation was enhanced through the inclusion of interactive events within the SCG module that required the users to provide information relevant to safety at the model mine site, and assess and understand issues that arise when working at heights. The value of the interaction within the module and the learning gained from interaction with it was enhanced through the development and use of modern instructional design techniques.

An evaluation of the integration of the module into the training program was performed and provided some useful insight into the integration of simulation into a training environment both off-site and subsequently onsite 
as discussed in the second paper. The integration also revealed some interesting issues and demonstrated the need for a cultural change with respect to the integration of SCG simulation into training activities and the need for ownership to be developed in subsequent projects.

Unfortunately the 2008 global financial crisis had an impact upon the ability of some of the commercial partners to engage in the final stages of the project. The project was brought to a successful conclusion and work in the area continues among the participants.

The conclusions and recommendations derived from this part of the study are summarised and discussions on the improvement of the SCG based simulation module are presented. Scientific testing of the module and its site deployment are presented in the second paper.

\section{Project structure}

The project resulted from industry and academic discussions about applying SCG simulation to mine workers' on-site inductions. An objective was to provide a pilot surface mine visualisation that enabled the concept to be demonstrated in context to industry and subsequently used for education and training. The SCG simulation would thus be directly relevant to the working conditions of a surface mine site in Australia. With some modification it would be relevant to surface mines globally.

Another objective was to bring together several collaborators with broad experience and knowledge to increase the project capability. This was a contractual and logistical challenge, but also very rewarding due to diversity in the project steering committee. Each collaborator had its own agenda but all had the common goal of improving safety on mine sites. The steering committee was the key project component where each party could present their position during the project life and allowed the project to be kept within scope. Using a steering committee can sometimes be cumbersome, but the overall result is very favourable and democratic, and the outcomes achieved in this case represented a balanced representation of the goals of the participants.

Figure 1 provides a schematic of project complexity and how the project management structure was built upon collaborative negotiation, leading to a formal project contract. Contracts took several months to complete and agreement was the result of compromise. This level manages collaborator expectations and ensures that scope of work, intellectual property, licensing and so on, are transparent and understood by all. On agreement, the project passed to the project directors who initiated the primary communications channels shown in Figure 1.

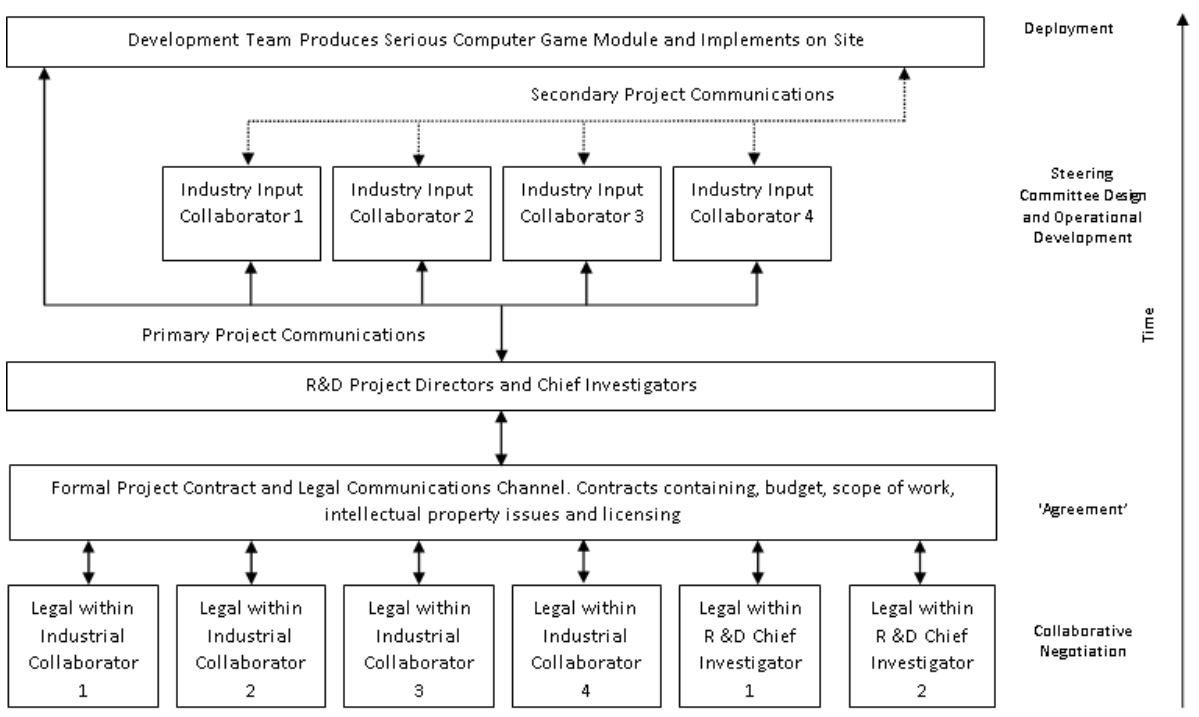

Figure 1. Foundations for collaborative module development. 
Effective communication was fundamental to the project. As the project gained momentum, a secondary less formal communications channel was developed for information exchange and to enable general operations to be streamlined.

Figure 2 summarises the project lifecycle and shows the start point and collaborative structure forming the project foundations. Once these were agreed, the project cycle commenced which required ongoing liaison between industry collaborators and the project directors. Appropriate industry personnel were identified that had significant subject matter experience allowing a formal module specification to be developed. It should be noted that the scale of an SCG project in a pilot study form with many unknowns is difficult to quantify and the expectations of collaborators must be managed at this point and throughout the project. Under a commercial, as opposed to a research and development project, this area must be defined in significant detail. There is a potential risk that projects become too open ended with too many variables and that unrealistic expectations can be introduced.

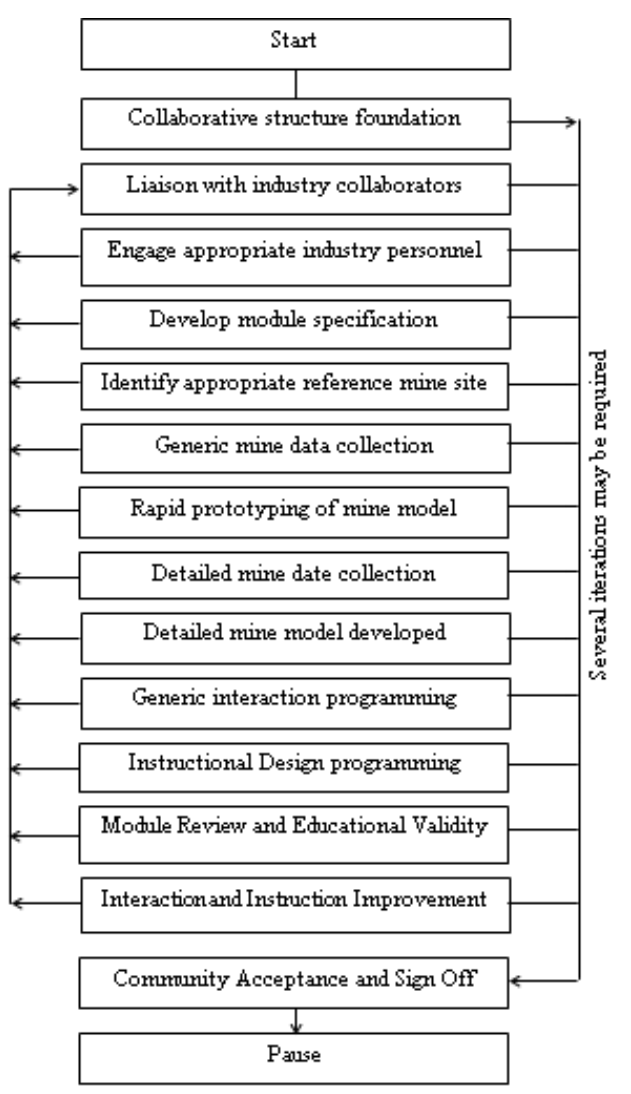

Figure 2. Module Development Cycle.

A reference mine site was selected and formed the model mine basis. Rapid prototyping commenced, followed by detailed on-site data collection and programming of generic interaction. Next was instructional design that is discussed in detail later in this paper and the second paper. The prototype was reviewed by the steering committee and a series of iterations was performed that brought the module to a point of community acceptance and sign-off. The exit point is represented as a 'pause' in Figure 2 because the pilot module was only a concept. Work is expected to continue.

As an aside, the challenges presented by this collaboration are pertinent to the wider sustainability of SCG in mining that is described by Stothard and Swadling [1] as lacking maturity, formal structure or direction when compared to other industries. In this project, there was a need for compromise between six competitive collaborators to achieve consensus and agreement that showed it is possible to achieve a common direction. This collaborative approach may be a basis for future projects that will address the lack of maturity in SCG 
simulation within the mining industry. The beginnings of this type of collaboration have begun with the Simulation Industry Association of Australia's Resources and Infrastructure Special Interest Group.

Working at Heights module selection

The Working at Heights subject area was chosen as the topic for the project module because it is identified as a potentially high risk area in mine site operation. The project steering committee consisted of members who were aware of the risks associated with working at heights and introduced the topic via the BHPB Fatal Risk Control Protocols [2] Chapter 9 of this comprehensive document is intended to eliminate or minimise the risk of fatalities, injuries and incidents arising from working at heights.

The Protocol applies wherever there is a potential for any person to fall $2 \mathrm{~m}$ or more, or to gain access to within $2 \mathrm{~m}$ of an open edge from where there is a potential to fall $2 \mathrm{~m}$ or more. The Protocol includes working from various forms of portable and moveable elevated work platforms, cages, ladders and scaffolding where objects could fall and cause injuries. The Protocol can also be applied through risk assessment where fall hazards from heights of less than $2 \mathrm{~m}$ are identified. The Protocol applies to all BHP Billiton controlled sites and controlled activities and to all employees, contractors and visitors. The Protocol covers in detail, reasons for inclusion of working at heights in the Protocol, requirements for plant and equipment, procedural requirements, people requirements and definitions.

The nature of the Working at Heights module is such that with modification, the module could easily be adapted to include protocols from other mine operators so that their protocols are represented. The module could also be used as a tool for collaboration to achieve best practice safety management.

Under this project, the Working at Heights module consisted of five sub scenarios that were intended as interactive visualisations of a generic surface mine site. The scenario related to risk assessment and the understanding of risk assessment procedures and hazard mitigation on site. The scenarios included an elevated work platform task, a ladders task, an excavation task and two scaffolding tasks. Task complexity varied between scenarios. The Working at Heights module began in the simulated classroom and takes the trainees through the necessary identification of personal protective equipment that must be used and also the correct documentation and procedures that must be followed before heading out on site. Trainees interacted with the simulation via question and answer sessions and were required to discuss issues in context with the trainers. Performance was monitored by the system and recorded to a database for later retrieval and review. Figure 3 is a screen shot of one of the scenarios.

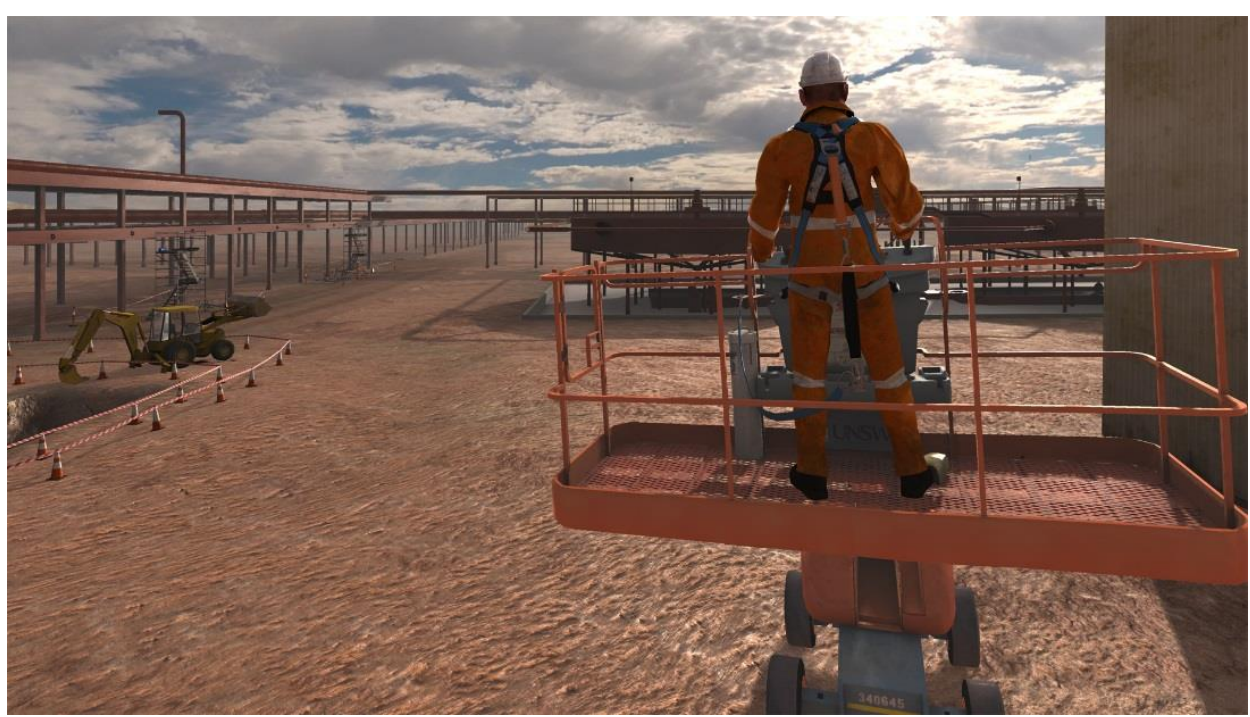

Figure 3. Screen shot of Working at Heights module. 
Serious computer games in mining

Serious computer game is a relatively new term that has resulted from the application of computer games technology to everyday or real life situations for visualisation, training and education. Many of the simulations already developed for mining applications have been based on computer games technology. A literature review was performed to identify SCG technology developed for the mining industry. The focus was research publications as a good indicator of activity, direction and current opinion on SCG. Table 1 summarises the results of the review.

AIMS Research investigated the use of virtual reality (VR) in mining and produced desktop PC VR hazard identification systems for mine safety training $[3,4]$. Squelch $[5,6]$ described VR for training mine workers that depicted hazards that a user must identify and address and remarked that a fully featured virtual reality simulator would be achieved through a multidisciplinary team consisting of, a computer programmer, mining engineer, training specialist, graphic artist and psychologist. Bise[7] noted that for VR to become a mine safety training tool, its attributes would have to be measured against other forms of delivery and developed virtual reality based miner training programs for hazard awareness. LeBlanc-Smith et al [8] report a VR application for integration of disparate three- and four dimensional (3D and 4D) mining data sets. Filigenzi et al [9] developed VR training software to reduce mining fatalities and built a mine simulation that presented hazards. Orr et al[10] continued development of the VR miner safety training that allowed mine workers to practice evacuation procedures.

UNSW performed an industry needs analysis for mining VR technology[11]. The project developed a hazard spotting simulations. The objective was to assist miners in the understanding of the risks, rules and procedures associated with coal mining. The project developed considerably over the 2000s[12-14]UNSW used off-the-shelf components, and developed a scalable system that could present simulations on the various classes of visualisation system described by Stothard et al[15]. The systems were tailored to the core competencies for mine workers and developed in collaboration with subject matter experts. Many detailed simulations were produced and instructional design and cognitive load theory was considered in detail [16, 17]. The long term objective was to achieve safe human responses so that mine safety can be improved.

Table 1 Simulation in mining papers.

\begin{tabular}{lll}
\hline Organisation/approximate date & Simulation & Purpose \\
\hline AIMS (1994, 1999) & $\begin{array}{l}\text { Underground and surface mine } \\
\text { operations and equipment } \\
\text { Underground goldmine stope model }\end{array}$ & $\begin{array}{l}\text { Visualisation, safety, design, risk management; } \\
\text { hazard identification and remediation }\end{array}$ \\
SIMRAC (1997, 2001) & Underground coal mine & Safety and hazard recognition \\
Penn' State University (1997) & Underground coal mine data & Web based disparate mine data visualisation \\
CSIRO $(1998,2000)$ & Underground coal mine & Hazard identification and remediation \\
NIOSH $(2000,2009)$ & Underground mine & Mine machinery, geology, geochemistry \\
MIRARCO (2003) & Surface environment & Hazard awareness \\
MISC (2008) & Underground hard rock & Tele-remote control \\
THALES (2004) & Operator training, & Equipment operation training and visualisation \\
Deutsche Montan Technologie GmbH (2004) & Underground platinum mine & Hazard identification and remediation \\
Tshwane University of Technology (2008) & Underground coal mining, surface & Visualisation, hazards training, safe work \\
UNSW (2001, 2004, 2006, 2007, 2009) & coal mining and uranium mining & $\begin{array}{l}\text { procedures, environmental and social impacts, } \\
\text { equipment training, site investigation }\end{array}$ \\
& Surface and underground mining & Visualisation, equipment operation, experimental \\
University of Queensland (2003) & & analysis, hazard spotting \\
\hline
\end{tabular}

Kizil [18] developed quality VR modules for the Minerals Council of Australia. The aim was improved visualisation and hazard identification training for mine workers. MIRARCO developed a VR lab system that visualised mine geoscience data and mine safety applications[19]. Swadling and Dudley[20] described a training simulation developed by Thales for a remotely operated load/haul/dump vehicle, in which operators could practise traversing the mine without risk of damage to the vehicles or mine. Statistics were gathered within the simulation to assist with operator assessment.

Deutsche Montan Technologie GmbH developed VR technology to train mine workers[21]. A VR training room was built and photorealistic depictions of various machines operating in an environment that allowed trainees to study and participate in an interactive man/ machine process as part of a group. The experience showed that 
a considered and systematic approach to operator training through the introduction of $\mathrm{VR}$ at a colliery produced significant improvements in operator training.

Van Wyk and DeVilliers[22] assessed the useability of VR based systems developed to train South African miners and referred to International Standards ISO 1997, ISO 9241-11 and ISO 1999, ISO 13407 for useability analysis. A VR training system was subsequently developed following interviews with mine managers.

Lucas et al[23] described the development of a VR simulation of a mining conveyor belt. The instructional based prototype was designed to familiarise the trainee with the working environment around the conveyor belt and alert them to the related hazards.

The above chronology of SCG in mining shows that many simulations have been developed at various key locations but that they have been developed in isolation. Bringing together a team of collaborators based in Australia has provided a baseline from which more complex international projects may be developed.

Serious computer game selection

Significant effort went into producing the 3D mine model, with the intention being to place as much information as possible into the model and module itself. The module was built using the popular 3D modelling packages 3D Studio Max and Maya.

Rapid interaction prototyping under the pilot project was via the Virtools game engine so that the existing capability at UNSW could be leveraged and so that the pilot module could be shown on scalable visualisation platforms ranging from a 360 degree fully immersive system, down to a mobile laptop computer web based application. However, a genuine concern of the steering committee was the cost and accessibility of certain game engines for some market sectors and as a result, a review of modern game engines was conducted to identify substitute platforms that may be more suited to mass market distribution and reduced runtime licensing costs[24].

The literature review showed that SCG based mining simulation has existed for some considerable time and been applied to education and training, it was also clear that many different game engines were available for the simulation application interface. Many different engines had been used and making the right choice was difficult with different drivers, such as financial or existing facilities for example, resulting in different engines being used.

Stothard et al[11]and Stothard and Galvin[25] performed industry needs analyses to identify SCG technology suitable for the NSW Coal Mining industry. A key outcome was that simulations should be easy to update and designed so that they could be built and updated by 'anyone' and not have to rely on specialist proprietary services. Hence drag and drop software was selected so that non-technical personnel could rapidly develop content for SCG applications. For the NSW coal mining industry, SafeVR and later Virtools were chosen from a plethora of VR development environments and games engines. Other researchers such as Schmid and Rossmann[26] used VRML, Lucas and Thabet[23] used Deep Creator and Orr et al[10]used the Unreal game engine. Eves and Meehan[27] reported that there were potentially hundreds of SCG engines available and choosing an appropriate engine was difficult.

Eves and Meehan[27] identified 12 selection criteria that considered current technology, costs, ongoing support costs, code versus runtime, documentation, support groups, sound support, networking, animation support, physics and editor tools. These were further classified as being essential, highly desirable and desirable and were used to reduce available engines from 280 to 10 possibilities. Detailed assessment criteria were then defined and the short listed game engines assessed. The study indicated that the Torque Game Engine represented the most complete environment and subsequent experiments revealed a 'low scoring' engine OGRE to be readily extendible to meet the project needs. Eves and Meehan[27] conclude that more consideration was required to establish a complete development environment.

Stothard and van den Hengel[24]conducted a comprehensive review of game engines under the Working at Heights project. An advantage of this review was that the UNSW and ACVT teams had significant experience in developing mining simulations and utilising SCG and were able produce a specific 'wish list'. There was also the 
added advantage that the teams had a known benchmark from which to consider and critique other SCG engines through practical application to mining SCG simulation, thus providing a point of reference towards future capabilities and requirements. The reviewed engines were placed into three categories: Open Source, Commercial and AAA. The AAA rating means commercially accessible, high license cost, high quality with a large user community.

The priority features identified for SCG mining simulations were: prototyping and rapid application development: smooth integration of 3D assets: ability to customise graphics pipeline using Shader languages (HLSL, GLSL, CG, etc.): support for multiuser simulations: support for web deployment: allowing collaboration between developers: reasonable cost and licensing agreement: ability to run across a cluster of computers and/or non-standard display; and product support/ community size.

The reviewed engines are shown in Table 2.

Table 2 Game engines reviewed in detail

\begin{tabular}{|c|c|c|}
\hline $\begin{array}{l}\text { Engine } \\
\text { category }\end{array}$ & Engine name & Comments \\
\hline \multirow[t]{6}{*}{$\begin{array}{l}\text { Commercial } \\
\text { engines }\end{array}$} & Virtools & $\begin{array}{l}\text { Comprehensive visual scripting language based authoring package (UNSW development } \\
\text { team had over } 3 \text { years experience using this package at an exceptionally high level.) }\end{array}$ \\
\hline & Quest 3D & Visual scripting languagebased authoring package, with similar features to Virtools. \\
\hline & Unity & $\begin{array}{l}\text { Inexpensive games engine with good 3D asset integration. Recently added Windows and } \\
\text { iPhone support }\end{array}$ \\
\hline & Torque & Ageing game engine with distributed networking capabilities \\
\hline & C4 Engine. & Inexpensive games engine with good licensing agreement. Supports COLLADA \\
\hline & $\begin{array}{l}\text { Virtual Battle } \\
\text { Space }\end{array}$ & $\begin{array}{l}\text { Military based simulation engine currently used by the Australian Defence Force. Increasing } \\
\text { in popularity among the serious games community }\end{array}$ \\
\hline \multirow[t]{3}{*}{ AAA engines } & Unreal Engine 3 & $\begin{array}{l}\text { Epic's major award winning game engine. Used both by Epic and a large number of third } \\
\text { party developers }\end{array}$ \\
\hline & CryENGINE 2 & $\begin{array}{l}\text { CryTek's next-gen engine used in Crysis. High system requirements, but generally recognised } \\
\text { as a leader in real time rendering research and technology. Growing popularity amongst } \\
\text { academic/serious games community }\end{array}$ \\
\hline & $\begin{array}{l}\text { Source Engine } \\
\text { ID Tech } 4 .\end{array}$ & $\begin{array}{l}\text { Valve's game engine used for Half Life } 2 \text { series. Ageing, but still popular. } \\
\text { Pioneering game engine of its time used for Doom3 }\end{array}$ \\
\hline $\begin{array}{l}\text { Open source } \\
\text { engines }\end{array}$ & $\begin{array}{l}\text { Panda } 3 D \\
\text { OGRE } \\
\text { Nebula } 2 \\
\text { Crystal Space } \\
\text { Irrlicht Engine }\end{array}$ & $\begin{array}{l}\text { Carnegie Mellon University's free Python based engine. Used by Disney for a variety of projects } \\
\text { Object oriented Graphics Rendering Engine. Available in several programming language bindings } \\
\text { Radon Labs' game framework. Used by several European commercial games } \\
\text { Popular object oriented game engine } \\
\text { Game engine supporting COLLADA format }\end{array}$ \\
\hline
\end{tabular}

It was found that for SCG mine simulation development, Virtools and Quest 3D were well rounded. Key components missing in Unity, Unreal and OGRE were cluster support and for Unreal and OGRE the lack of web deployment support was a problem. Web deployment is considered fundamental for scalable simulations that can leverage all classes of computer visualisation technology and content as described by Stothard et al[15]. Web deployment is also essential for making simulations available to the widest possible audience at relatively low cost. If web based deployment was the primary method of distribution then Unity or OGRE would be very favourable. Unity recently announced support for Windows and the iPhone platform. With its low cost, good file format support and multiple deployment platforms across single computers/processors it is well suited to the casual games community. Taking price as a priority consideration, quality, development time and flexibility of the game engines reviewed, as with Eves and Meehan,[27] OGRE may be the best SCG game engine to use for developing mining SCG simulations across different platforms. Unity also has potential and must be seriously considered. The recommendation for site based simulations was OGRE or Unity, with Virtools recommended for large multiscreen systems until cluster capability is proven in other engines.

\section{VideoTrace and module content}

One of the concerns in applying simulation technologies to industrial training is the lack of capability for end user editing of content. Real industrial environments are constantly evolving, and safety training needs to evolve similarly in order to maintain relevance and efficacy. The objective of the VideoTrace modelling section of the project was to evaluate the potential for the use of image based modelling as a technique for user creation of 3D content. 
VideoTrace is an image based modelling package[28]. To generate a 3D model that might be incorporated into a simulation the user captures a video sequence of the object to be modelled using an ordinary commercial video camera. Within VideoTrace the user then traces the shape of the object they wish to model. Through a 5 to 10 min tracing exercise a user with no modelling experience can create an accurate 3D model of an object from a video sequence. VideoTrace thus offers the prospect of separating the content creation process from the simulation technology, thus allowing a more flexible and user mediated approach to SCG. The development of the module leveraged manufacturer drawings, site drawings, photographs, videos and access to equipment hire companies. Collecting and collating this information and building models from it is very time consuming and this is where the issues of a clear specification for module development are required for any commercial activity and where VideoTrace can be used effectively.

The development team produced a fully interactive 3D model of the mine site environment to a high level of resolution and fidelity. However, model development time has always been a concern and the development of 3D models for the population of virtual reality or games is labour intensive and hence a general evaluation of the University of Adelaide's ACVT VideoTrace software was performed from a developer and user perspective.

The outcome of these investigations was that VideoTrace is potentially a very powerful and fast tool for producing 3D models and although the study found limitations in its application and that some areas required further development work, the models produced to populate the model for level of detail and scene complexity were very good. One project recommendation was that ACVT's VideoTrace software development continues as a matter of urgency.

Module integration and evaluation

Module integration and evaluation was approached from two angles. The first was an experimental site deployment to establish where the simulation module would fit into a training course in a formal deployment. The second was a series of controlled scientific experiments to collect and record data about the module with respect to the module's educational design. The experiments looked at improving the instructional design within the module and acquired user feedback as a process of ongoing improvement. The educational psychology experiments are discussed in detail in the second paper.

The pilot module was installed at a training facility in Adelaide, where the objectives were to develop a lesson plan that included the simulation, expose the module to a 'real' audience, test usability from a trainer and trainee perspective, acquire trainee demographic data, pre-training knowledge data, post-training knowledge data, training feedback data, simulator course material feedback, trainee's and trainer feedback and identify future improvements.

The module was displayed in a classroom environment on a $263 \mathrm{~m}$ projection screen. Over four days a group of 42 apprentices were exposed to the SCG simulation as part of the training process. Trainees were able to answer SCG simulator questions in real time on the screen via an individual keypad linked to a data record. The classroom approach was analogous to that of Schmid and Rossmann[29], Arggarwal et al[30]and McMaster et al[31]. Five simulator scenarios relating to 'Working at Heights' was presented to the trainees across the course of each day. Owing to time constraints and logistics, only the ladders scenario was used to collect data. Twenty people, split into two groups, were exposed to the simulation and 22 people split into two groups were exposed to the PowerPoint. The ladders module requires the trainees to use ladders and associated safe work procedures to repair a light globe on site when no elevated work platform is available. The module at this stage was instructor led.

Demographic data revealed that the participants were primarily Australian with English as their first language. Forty one of the participants were male and one was female. The oldest apprentice was 57 , the youngest was 26. Median age was 39. Median computer literacy across the groups was four out of five suggesting the majority would have no problem understanding text and verbal based instructions within the SCG simulator. However, if the module was used to train non-English speaking people from a different cultural background, it is fair to say that the module would need to be tailored to their language. The method of communication of ideas within the simulation may also need to be significantly different to cater for social and cultural differences. 
Before undertaking training, each participant completed a pre-training knowledge test on the safety issues and procedures surrounding ladders and the changing of a light globe. Following training, they completed a posttraining knowledge test to establish if any knowledge had transferred. The trainees were mature apprentices who had some mechanical and electrical background. The pre- and post-knowledge tests were aimed at identifying their level of knowledge relating to safe work procedures on mine sites, identification of risk and the use of safety equipment. The results of the pre- and post-training knowledge tests are presented in Figures 4 and 5 and show that there was an increase in knowledge for 38 out of the 42 trainees with respect to the learning outcomes of the 'light globe change using ladders' scenario across the Simulation groups A and B and the PowerPoint groups C and D.

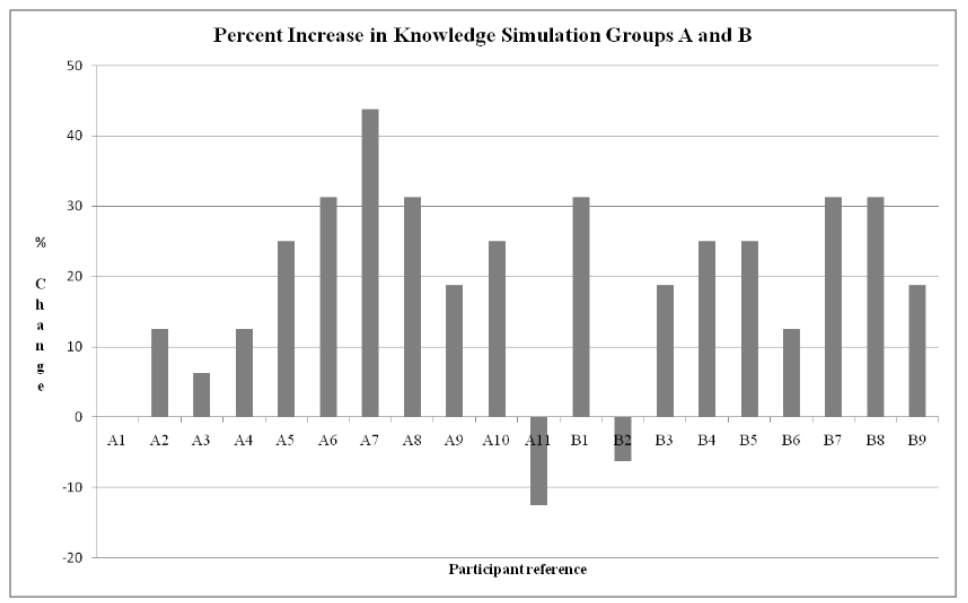

Figure 4. Per cent increase in knowledge in Simulation groups A and B.

The scores in the pre-knowledge test in Simulation groups A and B are shown in Table 3 . The data show that the trainees had slightly improved their knowledge through the simulation training session with the average score increasing from 52.50 to 68.75 . The standard deviation was reduced in the post-knowledge test suggesting focussing of knowledge among the trainees. However, it was unclear if this was actually due to the simulation because the simulation was part of the broader lesson plan. No trainees attained $100 \%$ and the increased Average may not show a sufficient transfer of knowledge through this method of training. Two of the 20 participants received 'negative' training - that is, their scores were worse in the post-knowledge test. These were subjects $A 11$ and $B 2$ who had a high computer literacy. The reason for negative training is unknown at this stage.

The scores in the pre-knowledge test in PowerPoint groups $C$ and $D$ are shown in Table 4. The data show that the trainees had only slightly improved their knowledge through the PowerPoint training session with the average score increasing from 50.52 to 59.38 .

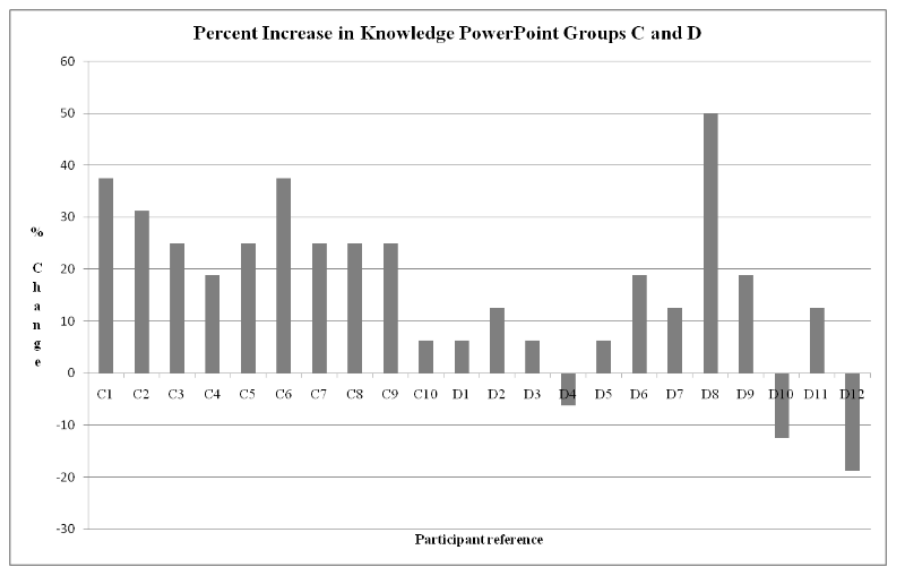

Figure 5. Per cent increase in knowledge in PowerPoint groups $C$ and D. 
The standard deviation was increased in the post-knowledge test suggesting a slight decrease in focussing of knowledge among the trainees. The data show that the trainees have only slightly improved their knowledge through the PowerPoint training session. However, again it is unclear if this is due to the PowerPoint at this stage as the PowerPoint was part of the broader lesson plan. None of the trainees attained $100 \%$. The Average score did increase but was less than the simulation groups and may show a limited transfer of knowledge through this method. Three of the 20 participants received 'negative' training - that is, their scores were worse in the postknowledge test. These were subjects D4, D10 and D12, who had a relatively high computer literacy. The reason for negative training is unknown at this stage.

In both the Simulation groups $A$ and $B$ and the PowerPoint groups $C$ and $D$, there was an Average increase in the knowledge between the pre- and post-knowledge tests.

\begin{tabular}{|c|c|c|c|c|c|}
\hline \multicolumn{6}{|c|}{ Group A and B (Simulator) } \\
\hline & & \multicolumn{2}{|l|}{ Pre-knowledge test } & \multicolumn{2}{|c|}{ Post-knowledge test } \\
\hline & & Score out of 16 & Per cent & Score out of 16 & Per cent \\
\hline \multirow[t]{11}{*}{ Group A } & $\mathrm{A} 1$ & 13.00 & $81 \cdot 25$ & 13.00 & $81 \cdot 25$ \\
\hline & A2 & 7.00 & 43.75 & 9.00 & 56.25 \\
\hline & A3 & 9.00 & 56.25 & 10.00 & 62.50 \\
\hline & $\mathrm{A} 4$ & 8.00 & $50 \cdot 00$ & 10.00 & 62.50 \\
\hline & A5 & 8.00 & $50 \cdot 00$ & 12.00 & 75.00 \\
\hline & A6 & $6 \cdot 00$ & 37.50 & 11.00 & $68 \cdot 75$ \\
\hline & A7 & $7 \cdot 00$ & 43.75 & 14.00 & 87.50 \\
\hline & A8 & 7.00 & 43.75 & 12.00 & 75.00 \\
\hline & A9 & 9.00 & $56 \cdot 25$ & 12.00 & 75.00 \\
\hline & A10 & 7.00 & 43.75 & 11.00 & $68 \cdot 75$ \\
\hline & A11 & 14.00 & 87.50 & 12.00 & 75.00 \\
\hline \multirow{13}{*}{ Group B } & B1 & 8.00 & $50 \cdot 00$ & 10.00 & 62.50 \\
\hline & B2 & $10 \cdot 00$ & 62.50 & 12.00 & 75.00 \\
\hline & B3 & 7.00 & 43.75 & 10.00 & 62.50 \\
\hline & B4 & 6.00 & 37.50 & 10.00 & 62.50 \\
\hline & B5 & 8.00 & $50 \cdot 00$ & 11.00 & 68.75 \\
\hline & B6 & 9.00 & $56 \cdot 25$ & 11.00 & 68.75 \\
\hline & B7 & 9.00 & $56 \cdot 25$ & 11.00 & 68.75 \\
\hline & B8 & 7.00 & 43.75 & 9.00 & $56 \cdot 25$ \\
\hline & B9 & 9.00 & 56.25 & 10.00 & 62.50 \\
\hline & & Maximum & 87.50 & Maximum & 87.50 \\
\hline & & Minimum & 37.50 & Minimum & $56 \cdot 25$ \\
\hline & & Average & 52.50 & Average & 68.75 \\
\hline & & Standard deviation & 12.56 & Standard deviation & 7.91 \\
\hline
\end{tabular}

Table 4 PowerPoint group pre- and post-knowledge scores

\begin{tabular}{|c|c|c|c|c|c|}
\hline \multicolumn{6}{|c|}{ Group C and D (PowerPoint) } \\
\hline & & \multicolumn{2}{|l|}{ Pre-knowledge test } & \multicolumn{2}{|l|}{ Post-knowledge test } \\
\hline & & Score out of 16 & Per cent & Score out of 16 & Per cent \\
\hline \multirow[t]{10}{*}{ Group C } & $\mathrm{C} 1$ & 8 & 50.00 & 14 & 87.50 \\
\hline & $\mathrm{C} 2$ & 8 & 50.00 & 13 & $81 \cdot 25$ \\
\hline & C3 & 7 & 43.75 & 11 & $68 \cdot 75$ \\
\hline & $\mathrm{C} 4$ & 10 & 62.50 & 13 & 81.25 \\
\hline & C5 & 10 & 62.50 & 14 & 87.50 \\
\hline & $\mathrm{C} 6$ & 1 & $6 \cdot 25$ & 7 & $43 \cdot 75$ \\
\hline & $\mathrm{C} 7$ & 9 & $56 \cdot 25$ & 13 & $81 \cdot 25$ \\
\hline & $\mathrm{C} 8$ & 4 & 25.00 & 8 & $50 \cdot 00$ \\
\hline & C9 & 3 & $18 \cdot 75$ & 7 & 43.75 \\
\hline & C10 & 10 & 62.50 & 11 & $68 \cdot 75$ \\
\hline \multirow[t]{16}{*}{ Group D } & D1 & 8 & 50.00 & 9 & $56 \cdot 25$ \\
\hline & D2 & 7 & $43 \cdot 75$ & 9 & $56 \cdot 25$ \\
\hline & D3 & 10 & 62.50 & 11 & $68 \cdot 75$ \\
\hline & D4 & 9 & $56 \cdot 25$ & 8 & 50.00 \\
\hline & D5 & 9 & 56.25 & 10 & 62.50 \\
\hline & D6 & 5 & $31 \cdot 25$ & 8 & $50 \cdot 00$ \\
\hline & D7 & 11 & $68 \cdot 75$ & 13 & $81 \cdot 25$ \\
\hline & D8 & 5 & 31.25 & 13 & 81.25 \\
\hline & D9 & 5 & 31.25 & 8 & 50.00 \\
\hline & D10 & 7 & 43.75 & 5 & 31.25 \\
\hline & D11 & 11 & 68.75 & 13 & 81.25 \\
\hline & D12 & 10 & 62.50 & 7 & 43.75 \\
\hline & & Maximum & 68.75 & Maximum & 81.25 \\
\hline & & Minimum & 31.25 & Minimum & 31.25 \\
\hline & & Average & 50.52 & Average & $59 \cdot 38$ \\
\hline & & Standard deviation & $13 \cdot 61$ & Standard deviation & $15 \cdot 42$ \\
\hline
\end{tabular}


Simulation groups A and B gained the highest average increase in knowledge. However, this was only a very small increase and may not actually be due to the Simulation or the PowerPoint. It may be a result of, the trainer, group dynamics, the simulation, the PowerPoint, engagement, no-consequences for failure, boredom and so on. The data does show that there had been a very slight improvement and transfer of a small amount of knowledge, but this is a broad assumption and whether these people had actually received any training is debatable. Of more concern, is that five people across the Simulation groups and the PowerPoint groups received 'negative training' and their scores were worse after training.

A further concern is that three of the five people were in PowerPoint group D, suggesting that the issue may not be the simulation or the PowerPoint but some other factor. If negative training occurs, people may be at risk on site. Whereas in the case of the two people that experienced 'negative training' in the Simulation groups, one was in group A and one was in group B. This could again be due to different trainers, group dynamics, etc. and demonstrates the need to control all variables when performing training and experimental analysis and most importantly keep trainees engaged in their training experience so that the learning outcomes are achieved. This must be investigated further with respect to overall course design as there may be a danger that many training courses do not promote deep understanding or lead to competency when people work on site. The overall course instructional design may need to be reviewed. To acquire course and SCG simulator feedback, the trainees completed a questionnaire. Trainees' responses were sought to questions about the Trainer/Facilitator, Programme Content, Course Material, Overall and finally Trainee's Comments, and were ranked using a Likert Scale. The Likert Scale had disagree (0), neither agree or disagree (3) and agree (5) as the indicators and no numbers were shown to the participants. The averaged data for each group are shown in Fig. 5 and show that the Trainer ranks highest with Material and Programme Content next and finally Overall ranking lowest. The simulator specific statements and responses were as follows:

(i) Learning through the interactive simulator was enjoyable. Group average was 3.48 out of 5; standard deviation 1.01

(ii) The simulator training was attention grabbing. Group average was 3.43 out of 5; standard deviation 1.05

(iii) It was easy to follow what was being taught by the simulator. Group average was 3.90 out of 5; standard deviation 0.81

(iv) I would have preferred to control the simulator myself. Group average was 3.86 out of 5; standard deviation 0.99

(v) I prefer standard training over simulator training. Group average was 2.83 out of 5; standard deviation 0.84

The data collected relating to the simulator show that the apprentices thought that learning through the interactive simulator was enjoyable. They thought the simulator was attention grabbing. It was easy to follow what was being taught by the simulator, but the knowledge test results do not necessarily confirm this. They would have preferred to control the simulator themselves. They slightly preferred the standard training to the simulator training when presented in the training session format performed at Adelaide. In future experiments, it would be pertinent to focus directly on the simulation when collecting feedback as the data is difficult to accurately assess due to unknown variables mentioned previously. The trainees were also asked to comment further on the training. The questions, text responses and interpretations were as follows:

1. If you disagreed with any of the statements above, it would be appreciated if you could tell us why?

2. What did you find to be the most useful aspect of this training - why?

3. Was the virtual reality training method compatible with your learning style and preference?

4. Did the simulator test your pre-existing Working at Heights knowledge?

5. Do you think the simulator increases your Working at Heights knowledge?

6. Please describe your favourite part of the simulator training. 
7. Please describe your least favourite component of the simulator training

8. What changes would you like to see made to the SCG component of the training program?

9. Can you offer any suggestions for improvement to the training course in general?

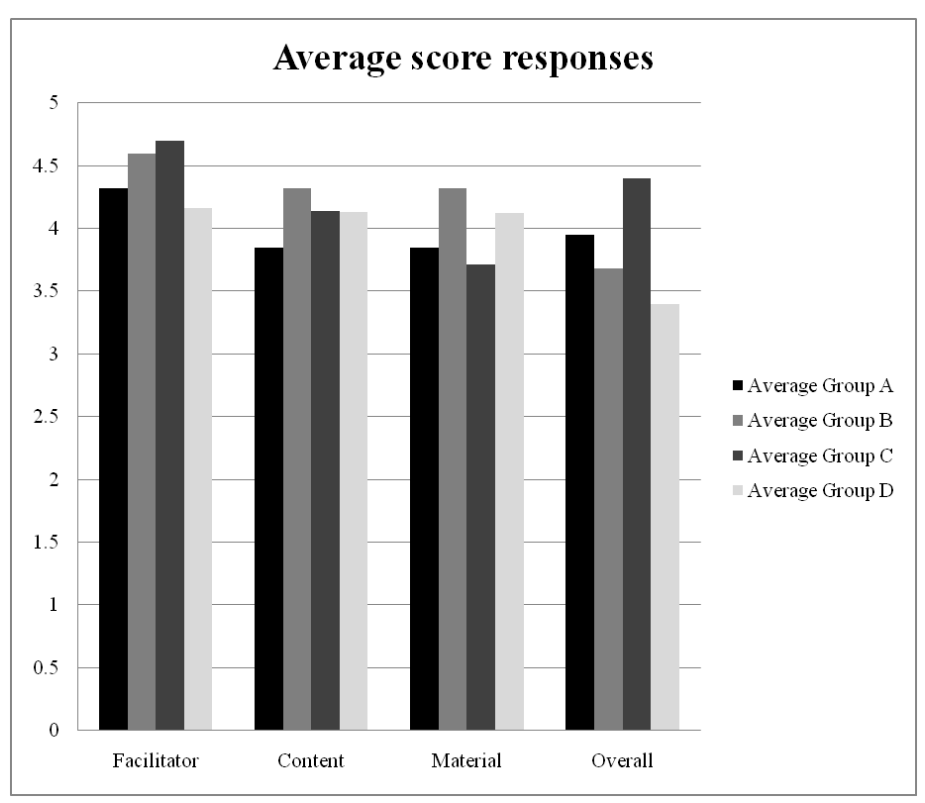

Figure 6 Trainee responses to course particulars.

Question 1 showed the apprentices would prefer to have a go on the simulator themselves and possibly for the simulation to be longer. They thought the simulation needed some improvement but it was quite real. Question 2 showed that the trainees particularly like the practical and hands on training part of the course, particularly the harness and using the equipment. This made sense as they are practical people. Hence, the simulation should probably be more practical and hands on too. The feedback from question 3 on whether the virtual reality simulation was compatible with the trainees' learning style was an overwhelming yes. Individual simulator training would be preferred by at least one trainee. The need for increased realism was commented upon too. Question 4 gained feedback on whether the simulation tested the trainees' Working at Heights knowledge and this was a clear yes. A couple of people realised that the simulation needed improvement and others remarked that they had no prior knowledge of Working at Heights. The feedback from question 5, on whether the simulation increased the trainees' Working at Heights knowledge was generally a yes. Some trainees thought that it only increased their knowledge a little. A comment was made on it being a good tool for the trainer to reinforce a subject. From question 6 , the trainees seemed to like visual aspects of seeing the mine site and understanding the context of the subject matter. Question 7 asked the trainees what they didn't like and clearly, they found the simulation too slow when operated by the trainer. Some of the functional processes like the image being obscured by the dialogues relate to the flat screen implementation and can be changed. Repetition in the questions was not popular either. The trainees would like to operate the simulation themselves. Question 8 showed that the changes that the trainees would like to see are more realism, a quicker simulation, increased screen size and possibly loaded on individual computers. The trainees would also like to see the simulator setup so that they can drive it themselves. Question 9 suggests that the consensus seemed to be that a larger screen size would make it more realistic and also the trainees should interact with the simulation themselves. The consequences of incorrect actions, was also considered important by one trainee.

The trainees provided some useful comments relating to the Working at Heights course and it is clear that these people are practical and want to do practical things. The transfer of knowledge during the session is a concern, but it must be remembered that this was the first iteration and the idea was to test the process and the training session format and the way that the simulation was presented was probably not ideal. This comment must be noted though because this may reflect an issue in the way training is delivered on site. The responses relating to the simulation suggest that the trainees want to use the system themselves and perform practical tasks within 
it. When sitting and observing the simulation, they found the simulation to be too slow and in some cases boring. The need for them to interact and engage with the material is extremely important. This may be where a remote off-site simulation can be developed that prepares trainees for onsite training. The trainees also did not really prefer the simulation training, suggesting in the form in which the simulation was presented at this stage of the project was not an ideal one. Trainee engagement with the simulation is something that early researchers of simulation comment upon in their deployment of simulators to the Mining industry where trainees must interacting with the system and not be passive observers.

\section{Discussion and conclusions}

This paper is the first of two papers that discuss the development of a serious computer game based training module and its integration into a mine site training program. The project was the result of a collaborative exercise that brought together a broad group of people that resulted in a rich serious game module based on a known industry risk, that is, Working at Heights. The module used the BHP Billiton Fatal Risk Protocols2 as a pointer for the subject matter area and as a result, a high quality SCG training module was developed. The project steering committee provided direction to the development of the module and its interaction, and the module was tested in a prototype form during a Working at Heights course presented to a group of apprentices in Adelaide before its formal deployment at the mine site. The data collected from the prototype deployment showed that the instructional design within the module and the manner in which it was integrated into training operations required considerable work to improve its suitability and sustainability. Also, when testing the module, the outcomes from trainees interacting with the module was not as good as expected and in fact showed that both the standard training course and SCG simulation module require significant work to improve their effectiveness. Some of the trainees' experienced negative training and most trainees did not appear to be engaged with the simulation. Negative training in conventional training scenarios has not been fully investigated and should be a serious consideration for further work. The effect of negative training will be addressed in the second paper via the application of modern instructional design techniques. The outcomes of the deployment lead to a scientific experiment being performed under controlled conditions and the instructional design of the module being improved prior to onsite deployment. This experiment led to some extensive modifications of the module and subsequent deployment of a much improved version, the details of which are reported in the second paper. The overall conclusions are that SCG modules can be developed via a collaborative framework and the technology used to develop the simulation can be tailored to a specific need. However, the design of the SCG must engage the end users with the visual and interactive content that they see on the screen. The trainees must be allowed interact with the module. The users of simulations in their training courses must also be engaged heavily in its design. This was the case in this pilot study, but consideration of instructional design must be made in much more detail for simulations to be integrated successfully into training programs. Under the prototype deployment reported here, it is reasonable to say that this did not happen. However, the trainees and trainers did respond to the simulation positively in some aspects and this was encouraging. Further work is required.

References

1. Stothard, P.M. and P. Swadling, Assessment of Maturity of Mining Industry Simulation. AusIMM International Transactions,Technical Note,, 2010. 119(Number 2): p. 102-109(8).

2. Anon, Fatal Risk Control Protocols (BHP Billiton). 2005: BHP Billiton.

3. Schofield, D., B. Denby, and McClaron, Computer Graphics in Virtual Reality in the Mining Industry. Mining Magazine, 1994.

4. Denby, B., et al., Hazard Awareness Training For Mining Situations Using 'Virtual Reality'. APCOM 98 Computer applications in the minerals industries. International symposium No27, London, 1998: p. 695-705.

5. Squelch, A., Virtual reality simulators for rock engineering related training, 1997, CSIR Mining Technology: New Delhi. 
6. Squelch, A., Virtual reality for mine safety training in South Africa. J. S. Afr. Inst. Min. Metall. SAIMM, Johannesburg., 2001. 101(4).

7. Bise, C.J., Virtual Reality: Emerging Technology for Training of Miners. Mining Engineering, 1997. 49(1).

8. LeBlanc-Smith, G., C. Caris, and G. Carter, Interactive Evaluation Of Mine Plans With Integrated Geological Exploration Data. Virtual Mine prototype: Interactive Visualisation Methods using VRML, Java and an Internet Browser., 1998, CSIRO.

9. Filigenzi, F.T., T.J. Orr, and T.M. Ruff, Virtual Reality for Mine Safety Training,. Applied Occupational and Environmental Hygiene, 2000. 15(6): p. 465-469.

10. Orr, T.J., Mallet, L. G., Klein, K.A.,, Enhanced Fire Escape Training for Mine Workers Using Virtual Reality Simulation. Proceeding of the Society of Mining Engineers. , 2009.

11. Stothard, P., et al., e-minesafe Safety and Training Simulator "The Integration of Knowledge and Skills to Achieve Safe Human Responses", 2001, UNSW School of Mining Engineering.

12. Stothard, P., J. Galvin, and C. Fowler, Development, Demonstration, and Implementation of a Virtual Reality Simulation Capability for Coal Mining Operations., in ICCR Conference2004, ICCR: Beijing, China.

13. Stothard, P. Developing and Deploying and Deploying Interactive Training Simulations for the Coal Mining Industry. in SIMTECT 2007 2007. Brisbane Australia: Simulation Industry Association of Australia.

14. Stothard, P., R. Mitra, and A. Kovalev. Assessing Levels of Immersive Tendency and Presence Experienced by Mine Workers in Interactive Training Simulators Developed for the Coal Mining Industry. in SimTect 2008 Conference Proceedings. 2008. Simulation Industry Association of Australia.

15. Stothard, P.M., et al. Taxonomy of Interactive Computer-based Visualisation Systems and Content for the Mining Industry - Part 1. . in Future Mining Conference. Proceedings. 2008. UNSW, Sydney, Australia.: AusIMM.

16. Bennett, L., P. Stothard, and J. Kehoe. Evaluating the Effectiveness of Virtual Reality Learning in a Mining Context. in SimTect 2010. 2010. Brisbane Australia: Simulation Industry Association of Australia.

17. Zhang, S., P.M. Stothard, and J. Kehoe, Evaluation of Underground Virtual Environment Training: Is the Mining Simulation or Conventional Power Point more Effective? , in SIMTECT SIMTECT, Editor 2010: Brisbane, Australia.

18. Kizil, M., A.P. Kerridge, and M.G. Hancock. Virtual Reality Applications in the Australian Minerals Industry. in 31st Int. Symposium on Computer Applications in the Minerals Industries Held under the auspices of the South African Institute of Mining and Metallurgy. 2003. Cape Town South Africa: SAIMM.

19. Delabbio, F.C., et al. The application of 3D CAD visualisation and Virtual Reality in the Mining and Minerals Processing Industry. HATCH, Mining Technology Unit, 2003.

20. Swadling, P. and J. Dudley, VRLoader - a Virtual Reality Training System for a Mining Application, 2004, Simulation Industry Association of Australia.

21. Schmid, M., Rossmann, M.,, Virtual Reality teaching and training systems in mining applications and operational experiences. Glauckauf Mining Reporter, 2004. 2.

22. Wyk, E.v. and R. DeVilliers. Virtual reality training applications for the mining industry. in Computer graphics, virtual reality, visualisation and interaction in Africa archive Proceedings of the 6th International Conference on Computer Graphics, Virtual Reality, Visualisation and Interaction in Africa. 2009. Pretoria South Africa: ACM New York USA.

23. Lucas, J. and W. Thabet, Implementation and Evaluation of a VR Task-Based Training Tool for Conveyor Belt Safety Training. ITcon 2008. 13: p. pp 654.

24. Stothard, P. and A. van-den-Hengel, Feasibility Study: Development and demonstration of virtual reality simulation training for the BHPB Olympic Dam Site Inductions, 2009, UNSW Sydney. 
25. Stothard, P. and J. Galvin, State of the Art of Virtual Reality Technology and its applications in 2005. 2005.

26. Schmid, M. and M. Rossmann, Virtual reality teaching and training systems in mining Applications and operational experiences. Glueckauf Mining Reporter,, 2004. 2: p. 39-44.

27. Eves, G. and P. Meehan. The Development of Selection Criteria for Game Engines in the Development of Simulation Training Systems. in Simtect 2008. 2008. Melbourne: Simulation Industry Association of Australia.

28. van den Hengel, A., et al. VideoTrace: Rapid interactive scene modelling from video. in ACM Transactions on Graphics. 2007.

29. Schmid, M. and T. Winkler. Interactive 3D supports underground repair and maintenance of road heading machines and shearer loaders. in Proceedings 21st World Mining Congress. 2008. Krakow.

30. Arggarawal, R., et al., An Evidence-Based Virtual Reality Training Program for Novice Laparoscopic Surgeons. Annals of Surgery, 2006. 244(2).

31. McMaster, L., et al. Combining 2D and 3D Virtual Reality for Improved Learning. in Proceedings of the International Society Society for Photogrammetry and Remote Sensing. 2008. Beijing. 